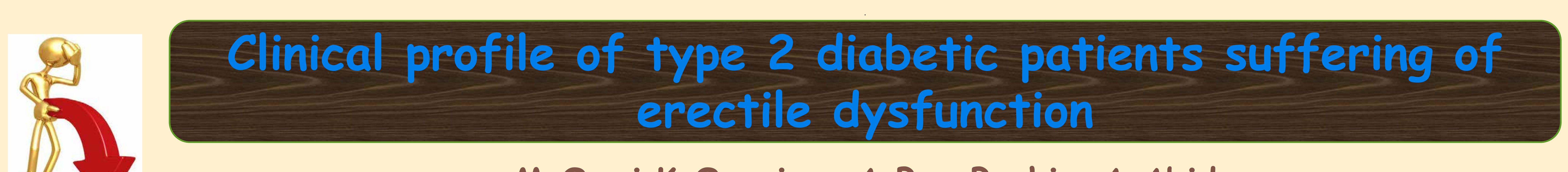

M.Omri,K.Ounaissa, A.Ben Brahim, A.Abid

EP531

Outpatient clinic and functional investigations

National Institute of Nutrition of Tunis, Tunisia

\section{IN RODUCFION}

oThe number of patients with diabetes has been increasing at an alarming pace for the last decade

$\checkmark$ Sexuality is frequently affected in diabetic patients.

While one man in five suffers from erectile dysfunction (ED)

in the general population, ED is 4.5 times more frequently observed in diabetic patients and starts 10 to 15 years earlier than in non-diabetics

\section{AlVS}

$\checkmark$ The aim of this study was to describe the clincal profile of a group of type 2 diabetic patients suffering of erectile dysfunction(ED).

\section{MEHHODOLOGY}

$\checkmark$ This was a cross sectional study involving a population of 70 type 2 diabetic patients recruited from the day hospital during May and June 2015.

$\diamond$ All men were invited to complete a sexual activity questionnaire (the abridged 5-item version of the International Index of Erectile Function-IIEF-5) as a diagnostic tool for ED.

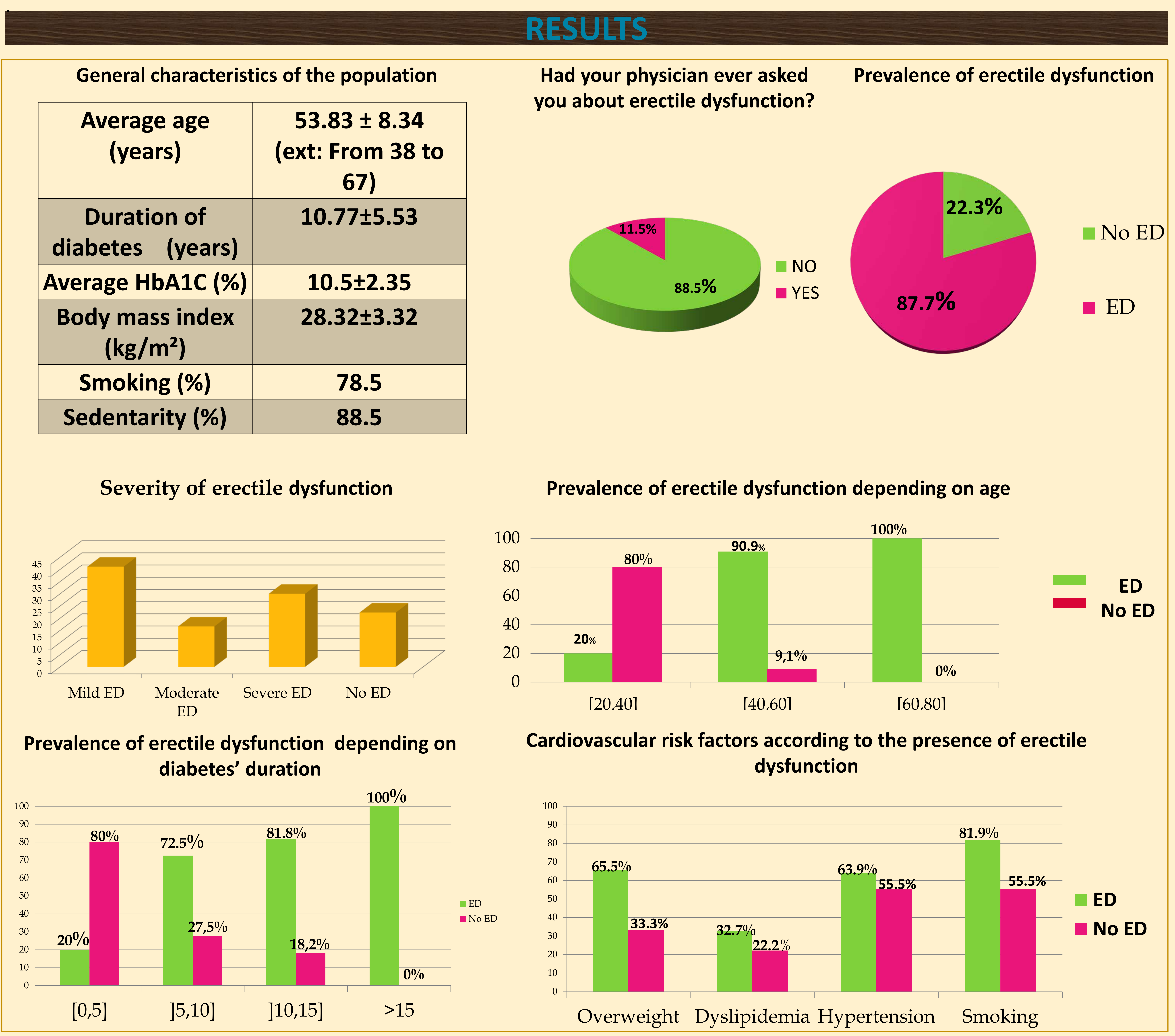

$\triangle F o r$ the majority of patients with diabetes, ED represents an important aggravation stage in the course of diabetes. $\triangle E D$ is indicative of the evolution of degenerative complications of diabetes. It is the most underdiagnosed of theses complications. This condition makes a significant impact on the personal and relational quality of life of patients. Consequently, ED should be subject to systematic screening 